\title{
Extended General Relativity for a Curved Universe
}

\author{
Mohammed. B. Al-Fadhli ${ }^{1 *}$ \\ ${ }^{1}$ College of Science, University of Lincoln, Lincoln, LN6 7TS, UK. \\ *Correspondence: malfadhli@lincoln.ac.uk; mo.fadhli7@gmail.com
}

\begin{abstract}
The Planck Legacy recent release revealed the presence of an enhanced lensing amplitude in the cosmic microwave background, which confirms the early universe positive curvature with a confidence level exceeding 99\%. Besides, the observed gravitational lensing within several galaxy clusters is higher than that estimated through the standard lambda cold dark matter model by an order of magnitude. While general relativity works perfectly well in the present universe where the spacetime is almost flat, it should be enhanced to account for the pre-existing universal curvature. This study presents new enhanced field equations utilising Einstein-Hilbert action. The enhanced field equations are reduced to Einstein field equations in a flat universe.
\end{abstract}

Keywords: General Relativity, Curved Universe.

\section{INTRODUCTION}

There is strong evidence of early universe positive curvature according to Planck Legacy recent release with a higher than $99 \%$ confidence level [1,2]. In addition, the detected gravitational lensing within several galaxy clusters by their substructures is higher than that estimated through the current standard model by an order of magnitude [3]. Enhancing general relativity by integrating the preexisting universal curvature might assist in resolving these inconsistencies.

This study presents new enhanced field equations based on Einstein-Hilbert action. The paper is organised as follows. In Section 2, the enhanced field equations are derived with considering the pre-existing universal curvature. Section 3 presents future works.

\section{New Field Equations}

The pre-existing universal curvature represented by the scalar curvature $\mathcal{R}$ is incorporated into Einstein-Hilbert action as

$$
S=\int\left[\frac{E_{D}}{2} \frac{R}{\mathcal{R}}+\mathcal{L}_{M}\right] \sqrt{-g} d^{4} x
$$

where $R=R_{u v} g^{u v}$ is the scalar curvature, $g=\operatorname{det} g_{u v}$ is the determinant of the metric tensor [4] and $E_{D}=$ (stress/strain) in $\left(\frac{N}{m^{2}}\right)$ is the deformation modulus reflecting the stress energy tensor of the matter in terms of the Lagrangian $\mathcal{L}_{M}$ divided over the change in spacetime curvature $R_{u v}$ with respect the pre-existing universal curvature $\mathcal{R}$. Using the principle of least action $\delta S=0$ of the variation of the action over the inverse metric tensor $\delta g^{\mu \nu}$

$$
\begin{gathered}
S=\int\left[\frac{E_{D}}{2} \frac{\delta\left(R \mathcal{R}^{-1} \sqrt{-g}\right)}{\delta g^{\mu \nu} \sqrt{-g}}+\frac{\delta\left(\sqrt{-g} \mathcal{L}_{M}\right)}{\delta g^{\mu \nu} \sqrt{-g}}\right] \delta g^{\mu \nu} \sqrt{-g} d^{4} x \\
S=\int\left[\frac{E_{D}}{2}\left(\frac{\delta R}{\mathcal{R} \delta g^{\mu \nu}}-\frac{R \mathcal{R}}{\delta \mathcal{R}^{2} \delta g^{\mu \nu}}+\frac{R \delta \sqrt{-g}}{\mathcal{R} \sqrt{-g} \delta g^{\mu \nu}}\right)+\frac{\delta\left(\sqrt{-g} \mathcal{L}_{M}\right)}{\delta g^{\mu \nu} \sqrt{-g}}\right] \delta g^{\mu \nu} \sqrt{-g} d^{4} x
\end{gathered}
$$

Eq. (3) have to hold at any variation in $\delta g^{\mu \nu}$ as

$$
\frac{\delta\left(R_{u v} g^{u v}\right)}{\mathcal{R} \delta g^{\mu \nu}}-\frac{R \mathcal{R}}{\delta\left(\mathcal{R}_{u v} g^{u v}\right)^{2} \delta g^{\mu \nu}}+\frac{R \delta \sqrt{-g}}{\mathcal{R} \sqrt{-g} \delta g^{\mu \nu}}=\frac{-2 \delta\left(\sqrt{-g} \mathcal{L}_{M}\right)}{E_{D} \delta g^{\mu \nu} \sqrt{-g}}
$$

The four terms in Eq. (4) are solved individually as follows 


\subsection{First Term}

The variation of the first term is

$$
\frac{\delta\left(R_{u v} g^{u v}\right)}{\mathcal{R} \delta g^{\mu v}}=\frac{R_{u v} \delta g^{u v}+g^{u v} \delta R_{u v}}{\mathcal{R} \delta g^{\mu v}}
$$

where $\delta R_{u v}$ is the variation of the Ricci curvature tensor and $\delta g^{\mu \nu}$ is the variation of the inverse metric tensor $g^{\mu \nu}$. The variation in the Ricci curvature tensor can be obtained by contacting over two indices in the variation of the Riemann curvature tensor $R_{\sigma \mu \nu}^{\rho}$ [5]. The Riemann curvature tensor is defined as

$$
R_{\sigma \mu \nu}^{\rho}=\partial_{\mu} \Gamma_{v \sigma}^{\rho}-\partial_{\nu} \Gamma_{\mu \sigma}^{\rho}+\Gamma_{\mu \lambda}^{\rho} \Gamma_{v \sigma}^{\lambda}-\Gamma_{\nu \lambda}^{\rho} \Gamma_{\mu \sigma}^{\lambda}
$$

where $\Gamma_{\mu \nu}^{\lambda}$ is the Levi-Civita connection. The variation of the Riemann curvature tensor is

$$
\delta R_{\sigma \mu \nu}^{\rho}=\partial_{\mu} \delta \Gamma_{v \sigma}^{\rho}-\partial_{\nu} \delta \Gamma_{\mu \sigma}^{\rho}+\delta \Gamma_{\mu \lambda}^{\rho} \Gamma_{\nu \sigma}^{\lambda}+\Gamma_{\mu \lambda}^{\rho} \delta \Gamma_{v \sigma}^{\lambda}-\delta \Gamma_{\nu \lambda}^{\rho} \Gamma_{\mu \sigma}^{\lambda}-\Gamma_{v \lambda}^{\rho} \delta \Gamma_{\mu \sigma}^{\lambda}
$$

where $\delta \Gamma_{v \sigma}^{\rho}$ represents the difference between two connections where its covariant derivative is

$$
\nabla_{\mu}\left(\delta \Gamma_{v \sigma}^{\rho}\right)=\partial_{\mu}\left(\delta \Gamma_{v \sigma}^{\rho}\right)+\Gamma_{\mu \lambda}^{\rho} \delta \Gamma_{\nu \sigma}^{\lambda}-\Gamma_{\mu \nu}^{\lambda} \delta \Gamma_{\lambda \sigma}^{\rho}-\Gamma_{\mu \sigma}^{\lambda} \delta \Gamma_{\nu \lambda}^{\rho}
$$

By substituting Eq. (8) to Eq. (7), the variation of the Riemann curvature tensor can be rewritten as

$$
\delta R_{\sigma \mu \nu}^{\rho}=\nabla_{\mu}\left(\delta \Gamma_{\nu \sigma}^{\rho}\right)-\nabla_{\nu}\left(\delta \Gamma_{\mu \sigma}^{\rho}\right)
$$

The variation in the Ricci curvature tensor $\delta R_{u v}$ can be obtained by contracting two indices in Eq. (9) and acquiring the Palatini identity [5] as

$$
\delta R_{\sigma v}=\nabla_{\rho}\left(\delta \Gamma_{\nu \sigma}^{\rho}\right)-\nabla_{v}\left(\delta \Gamma_{\rho \sigma}^{\rho}\right)
$$

Regarding the variation of the inverse metric tensor $\delta g^{\mu \nu}$, it can be obtained according to the metric compatibility of the covariant derivative, $\nabla_{\rho} g^{\mu \nu}=0$ and by renaming the dummy indices $v$ and $\rho$ to $\rho$ and $\mu$ respectively then substitute Eq. (10) as

$$
g^{\sigma v} \delta R_{\sigma v}=\nabla_{\rho}\left(g^{\sigma v} \delta \Gamma_{v \sigma}^{\rho}-g^{\sigma \rho} \delta \Gamma_{\mu \sigma}^{\mu}\right)
$$

To obtain the total derivative, Eq. (11) is multiplied by $\sqrt{-g}$ [5] where for any tensor density it is as follows

$$
\nabla_{\mu}\left(\sqrt{-g}\left(g^{\sigma v} \delta \Gamma_{v \sigma}^{\mu}-g^{\sigma \mu} \delta \Gamma_{\mu \sigma}^{\mu}\right)\right)=\partial_{\mu}\left(\sqrt{-g}\left(g^{\sigma v} \delta \Gamma_{\nu \sigma}^{\mu}-g^{\sigma \mu} \delta \Gamma_{\mu \sigma}^{\mu}\right)\right)
$$

Integrating Eq. (12) produces a boundary term according to Stokes theorem. The variation of the inverse metric tensor $\delta g^{\mu \nu}$ as well as its partial derivative $\partial_{\sigma} \delta g^{\mu \nu}$ do not vanish. Therefore, the boundary term exists. However, as the variation vanishes as it approaches the neighbourhood of the boundary, its contribution to the action can be discounted [5]. Therefore, the first term reduced to

$$
\frac{\delta\left(R_{u v} g^{u v}\right)}{\mathcal{R} \delta g^{\mu v}}=\frac{R_{u v}}{\mathcal{R}}
$$

where this term represents the change of spacetime curvature $R_{u v}$ over the pre-existing universal conformal curvature $\mathcal{R}$ 


\subsection{Second Term}

The variation of the second term is

$$
\frac{R \mathcal{R}}{\delta\left(\mathcal{R}_{u v} g^{u v}\right)^{2} \delta g^{\mu v}}=-\frac{2 R\left(\mathcal{R}_{u v} \delta g^{u v}+g^{u v} \delta \mathcal{R}_{u v}\right)}{\mathcal{R}^{2} \delta g^{\mu v}}
$$

According to the metric compatibility of the covariant derivative, $\nabla_{\rho} g^{\mu v}=0$ and by renaming the dummy indices $v$ and $\rho$ to $\rho$ and $\mu$ respectively then substitute Eq. (10) as

$$
g^{\sigma v} \delta \mathcal{R}_{\sigma v}=\nabla_{\rho}\left(g^{\sigma v} \delta \Gamma_{v \sigma}^{\rho}-g^{\sigma \rho} \delta \Gamma_{\mu \sigma}^{\mu}\right)
$$

Utilising Stokes theorem as with Eq.(12), the second term reduce to

$$
\frac{R \mathcal{R}}{\delta\left(\mathcal{R}_{u v} g^{u v}\right)^{2} \delta g^{\mu \nu}}=-\frac{2 \mathcal{R}_{u v} R}{\mathcal{R}^{2}}
$$

\subsection{Third Term}

Utilising the Jacobi's formula, the differentiation of the determinant and its variation are

$$
\delta g=\delta \operatorname{det}\left(g_{u v}\right)=g g^{\mu v} \delta g_{u v}, \quad \delta \sqrt{-g}=-\frac{\sqrt{-g}}{2} g_{u v} \delta g^{\mu v}
$$

where $g_{u v} \delta g^{\mu v}=-g^{\mu v} \delta g_{u v}$ and $\delta g^{\mu v}=-g^{\mu \alpha}\left(\delta g_{\alpha \beta}\right) g^{\beta v}$ [5]. Accordingly, the third term is

$$
\frac{R}{\mathcal{R} \sqrt{-g}} \frac{\delta \sqrt{-g}}{\delta g^{\mu \nu}}=-\frac{R}{2 \mathcal{R}} g_{u v}
$$

\subsection{Fourth Term}

The fourth term is proportional to the stress-energy tensor by the definition [6] as follows

$$
\frac{-2 \delta\left(\sqrt{-g} \mathcal{L}_{M}\right)}{\delta g^{\mu \nu} \sqrt{-g}}=g_{u v} \mathcal{L}_{M}-2 \frac{\delta \mathcal{L}_{M}}{\delta g^{\mu \nu}}:=T_{u v}
$$

\subsection{Enhanced Field Equations}

Substituting Eqs. $(19,18,16,13)$ to Eq. (4), generates the new field equations

$$
\frac{R_{u v}}{\mathcal{R}}+\frac{2 \mathcal{R}_{u v} R}{\mathcal{R}^{2}}-\frac{R}{2 \mathcal{R}} g_{u v}=\frac{T_{u v}}{E_{D}}
$$

The enhanced field equations are reduced to Einstein field equations in a flat universe that in which the curvature tensor through space and conformal time $\mathcal{R}_{u v}=0$ as well as the scalar curvature over the conformal time $\mathcal{R}=1$.

\section{FUTURE WORK}

The Newtonian limit to be taken for the enhanced field equations in future works. 


\section{References}

[1] E. Di Valentino, A. Melchiorri, and J. Silk, "Planck evidence for a closed Universe and a possible crisis for cosmology," Nat. Astron., vol. 4, no. 2, pp. 196-203, Feb. 2020.

[2] N. Aghanim et al., "Planck 2018 results. V. CMB power spectra and likelihoods," Astron. Astrophys., vol. 89, Jun. 2020.

[3] M. Meneghetti et al., "An excess of small-scale gravitational lenses observed in galaxy clusters," Science (80-. )., vol. 369, no. 6509, pp. 1347-1351, Sep. 2020.

[4] N. Straumann, “General Relativity (Graduate Texts in Physics)” in Springer, Springer, 2013.

[5] S. M. Carroll, "Spacetime and Geometry: An Introduction to General Relativity" 2003.

[6] S. M. Carroll, “Lecture Notes on General Relativity”, 1997. 